\title{
The Formal Structure of Ecological Contexts
}

\author{
Barry Smith ${ }^{1}$ and Achille C. Varzi ${ }^{2}$ \\ ${ }^{1}$ Department of Philosophy, SUNY Buffalo, NY 14260, USA \\ phismith@buffalo.edu \\ ${ }^{2}$ Department of Philosophy, Columbia University, New York, NY 10027, USA \\ achille.varzi@columbia.edu
}

\begin{abstract}
This paper presents the outline of a formal ontology of contexts. More specifically, it deals with the ontology of ecological contexts (niches, habitats, environments, ambients) and of the relations between organisms, niches, and the spatial regions they occupy. The first part sets out the basic conceptual background. The second part outlines a semi-formal theory which builds upon notions and principles of mereology, topology, and the theory of spatial location.
\end{abstract}

\section{Introduction}

The ecological literature distinguishes between two ways of conceiving a "niche" (habitat, ecotope, biotope, microlandscape) [22, 39]. On the one hand, there is the traditional functional conception of a niche as the role or position enjoyed by an organism or population within an ecological community. As C. Elton [14] famously put it, "When an ecologist says 'there goes a badger' he should include in his thoughts some definite idea of the animal's place in the community to which it belongs, just as if he had said 'there goes the vicar'." The world of niches might, in this sense, be viewed as a giant evolutionary hotel, some of whose rooms are occupied (by organisms which have evolved to fill them), some of whose rooms are for a variety of reasons unoccupied but can become occupied in the future. On the other hand, there is the environmental conception advanced by G. E. Hutchinson [19] and R. Lewontin [21]. On this second conception, a niche is thought of as the hypervolume defined by the limiting values of all environmental variables relevant to the survival of a given species. A niche is not a mere location, but a location in space that is defined additionally by a specific constellation of ecological parameters such as degree of slope, exposure to sunlight, soil fertility, foliage density, and so on. It is, we might say, an ecological context. The purpose of this paper is to outline a formal theory of this notion.

Our account expands on the theory put forward in [30], which builds upon certain fundamental notions and principles of mereology, topology, and the theory of spatial location. We focus on niche tokens, which is to say on the environmental niche determined by a given organism or population of organisms in a given place, and we aim to be more explicit than is customary in the ecological literature as concerns the

P. Bouquet, P. Brezillon, and L. Serafini (eds.), Modeling and Using Context. Proceedings of the Second International and Interdisciplinary Conference, Berlin and Heidelberg: Springer-Verlag, 1999, pp. 339-350. 
ontological marks of these entities. Thus, while our theory will be illustrated above all by means of simple ecological and biological examples, it should be understood as being applicable in principle to a wide range of different domains. The concept of niche and its cognates are indeed already employed ubiquitously in many disciplines, from sociology [15] and economics [23] to organization science [3]. Yet the underlying principles have thus far been investigated not at all from the formal point of view. This is in part because the mereotopological tools needed for such an investigation have been developed only recently. But it is in part also a consequence of the fact that formal ontologists have tended to shun holistic structures, preferring to conceive reality in terms of what can be simulated via (normally set-theoretic) constructions from out of postulated atoms or Urelemente. Our account, in contrast, is resolutely holistic: it proceeds from the idea that there are structured wholes, including the medium of space, which come before the parts that these wholes contain and that can be distinguished on various levels within them.

\section{General Background}

Our theory has two main progenitors. One is the Aristotelian theory of place, as sketched in the Physics [29]. Each body, in Aristotle's view, has a place, and the place contains the body-it relates to its body in something like the way the interior boundary of an urn relates to the liquid contained within it. Thus, a place exactly surrounds the body in such a way that the body is separate from but yet in perfect contact with its surrounding place, the latter being therefore marked by a certain sort of interior cavity or hole. An ecological context, in our sense, is like the place of the relevant organism or population, in Aristotle's sense.

The second progenitor is the account of settings elaborated in great detail by the ecological psychologist Roger Barker [1, 2, 25]. Consider, on the one hand, the recurrent settings that serve as the environments for the everyday activities of persons and groups of persons. Examples are: John's swimming pool, your favorite table in the cafeteria, the 7:50am train to Verona. Each of these settings is marked by certain stable arrays of physical objects and physical infrastructure (by 'surface layouts', in Gibson's [16] terms). But each recurrent setting is associated, on the other hand, with certain stable patterns of behavior on the part of the persons involved. Physicalbehavioral units are the conjunct of these two aspects. They are built out of both physical and behavioral parts. As Barker [1] puts it, they "are common phenomenal entities, and they are natural units in no way imposed by an investigator." Each physical-behavioral unit has thus two sorts of components: people behaving in certain ways (lecturing, listening, eating) and non-psychological objects with which behavior is transacted (walls, chairs, electricity, etc.). Each unit is marked by the opposition between an organized internal (foreground) pattern from a wider external (background) pattern [20]. And each is circumjacent to its components: the former surrounds (encloses, encompasses) the latter - the pupils and equipment are in the class; the swimmers are in the swimming pool. All of these are features that are shared by ecological contexts, as we understand them here. Moreover, ecological contexts, like physical- 
behavioral units, may be nested together in hierarchies. There are typically many units of each lower-level kind within a given locality, and these are typically embedded within larger units, as a game is embedded within a match. The same goes for ecological contexts.

It is somewhat remarkable that similar characterizations may be found in quite different domains of application. For instance, the notion of a mobile ambient employed in the theory of network security is close in spirit-ontologically - to that of a physical-behavioral unit. Cardelli and Gordon [4] characterize an ambient as "a bounded place where computation happens", with a boundary around it. Examples include: a web page (bounded by a file), a virtual address space (bounded by an addressing range), a Unix file system (bounded within a physical volume), a laptop (bounded by its case and data ports). "If we want to move computations easily we must be able to determine what should move; a boundary determines what is inside and what is outside an ambient." [id.]

Related ideas may be found also in the anthropological literature on territoriality, a phenomenon that arises whenever there obtains a type of relation between an individual or group and a structured area or volume of space which is of such a sort that the individual or group will seek to defend the latter against invasion by other conspecific individuals or groups [24]. (Compare also the related psychological phenomenon of 'personal space' [17].) Anthropologists have shown that, in the case of both human and non-human animal species, a nested hierarchy of types of site must be distinguished around any given individual or group. The force of territoriality then diminishes with increase in group size and spatial area. In the first place there are territories in the narrow sense, the characteristically tiny areas in relation to which the occupying individual or group demands exclusive use. This central area is then extended to comprehend various attached regions, for example watering holes, where desirable resources are available on a routine basis. Finally we have the home range, that larger surrounding area within which the group spends almost all of its time [33]. Again, we find here the idea that niches (territories, settings) form a nested hierarchy around an individual or group occupying a privileged locus at its center.

Let us, then, summarize the ontological marks of niches, understood as genuine ecological contexts. (i) A niche is not simply a location in space; rather, it is a location in space that is constrained and marked by certain functional properties (of temperature, foliage density, federal jurisdiction, etc.). (ii) A niche occupies a physicaltemporal locale within which is a certain privileged locus-a hole-into which the relevant organism fits exactly. (iii) A niche is a connected whole with a more or less determinate outer boundary: there are things that fall clearly within it, and other things that fall clearly outside it. (iv) A niche may have parts that are also niches, and a niche may similarly be a proper part of larger, circumcluding niches. (v) A niche may overlap spatially with other niches (of different organisms) with which it does not share common parts. As we said, these ontological marks correspond to the environmental notion of niche-the concrete (token) ecological context that is actually occupied by a given organism or group of organisms on a given occasion. We may nonetheless assume that functional niches, too, to the extent that they are realized at all, are realized in (or as) some concrete environmental niches or habitats of the sort considered here. 


\section{The Formal Theory}

We shall now lay out the formal principles underlying this characterization. As in [30], we shall initially suppose that all tenants are compact, in the sense that they have no interior cavities. Later we shall see how the account can be extended to the case of tenants with one or more cavities. Note that our principles are to be given a tensed reading: we are concerned here with the panoply of niche-tenant relations at a given time.

The first axiom fixes the basic spatial relationships between niches and their tenants. Formally, a niche is a certain type of neighborhood-a perforated or deleted neighborhood of its tenant. Thus, we require that a niche should not overlap but rather surround its tenant:

\section{A1 If $x$ is a niche for $y$, then $x$ surrounds $y$.}

This implies that a niche is disjoint from its tenant, not only in the mereological sense of not sharing any part with the niche, but also in the purely spatial sense of not sharing any common location. This is important, because we must in the present context draw a clear distinction between those mereological (part-whole) relations that apply to a given set of spatial entities and those that apply to the spatial regions those entities occupy [7, 35]. It is a characteristic property of physical objects that they may not be in the same place at the same time. But this principle does not hold when entities of other kinds are countenanced. For instance, we want to say that the region where a niche is located may be occupied by objects that are not a part of the niche. The niche around the sleeping bear may be full of flies, but it need not be the case that the flies themselves are a part thereof. Moreover, the tokened environmental niches which form the center of our theory are bounded not just spatially, and not just via physical material (the walls of the cave), but also via thresholds in quality-continua (for instance, temperature). Distinct niches, therefore, may occupy the same or overlapping spatial regions, and different organisms, or organisms of different types, may be able to find niches within the same spatial region without its thereby being implied that they share a niche. A niche for the fly on the bear's nose is not a part of the niche for the bear (or at least: we need not assume that it is), though it overlaps spatially therewith.

As a second axiom, we require that a niche be in contact with its tenant (that nothing can squeeze in between them, as it were):

\section{A2 If $x$ is a niche for $y$, then $x$ is connected to every tangential part of $y$.}

A tangential part is, of course, a part that is connected to the spatial complement of the relevant entity. And the relevant notion of contact is to be understood along the lines of classical topological connection: two entities are connected if they share at least a boundary. More precisely, there must be a common boundary which is part of one or the other, though not necessarily of both [5,27]. This notion if connection is not unproblematic [28, 37], and there are ways of characterizing a relation of topological connection which do not rely on the boundary concept at all [10, 11]. Here, however, we shall content ourselves with this ordinary account. In particular, we shall assume that two discrete entities can be in contact only if one of them is open (i.e., if 
it does not include its boundary) where the other is closed (in the sense that it includes its boundary as a proper part). Thus, if John and Mary are topologically closed, then genuine contact between them is impossible if contact is understood in terms of topological connection. Due to the density of space, the surfaces of two distinct physical bodies cannot be in contact topologically, though they may of course be so close to each other that they appear to be in contact to the naked eye. (This is in agreement with ordinary topology, and also with standard physics, but see $[26,31]$ for more details on the underlying issues.)

Note indeed that A1 implies that a niche is always externally connected to its tenant: they are connected, but they do not overlap. Since nothing is externally connected to itself, it follows immediately that the niche-tenant relation is irreflexive. Moreover, note that A2 depends crucially on our supposition concerning the compactness of all tenants. The presence of an inner cavity would split the tangential parts of the tenant into two classes, but A2 is only meant to apply to those tangential parts that face outwards, as it were. We shall come back to this point in the next section.

Our third axiom constrains the topology of niches by ruling out the possibility that they be spatially scattered:

\section{A3 If $x$ is a niche for $y$, then $x$ is self-connected (i.e., in one piece).}

Again, the relevant notion here is to be understood in classical topological terms: $x$ is self-connected if and only if any two parts that make up $x$ are connected to each other [5]. Thus, every niche must enjoy a certain natural completeness or rounded-offness. Note that we do not assume a corresponding principle concerning the topological structure of a tenant. In agreement with standard ecological treatments, a tenant may be a single organism or a population of separate organisms. There is, for instance, a natural niche surrounding John and Mary as they enjoy a romantic candle-light dinner. For another example, avatars (a shoal of fish in a lake, a herd of buffalo) are causally integrated and more or less reproductively isolated subpopulations of conspecifics, and their identity conditions typically involve reference to a relevant ecological context $[12,13]$. Avatars play an important role in evolutionary theory in light of the fact that it is avatars, and not whole species, that are the most plausible candidate subjects of selective pressures at the group level.

A constraint on the topology of tenants comes from our fourth axiom, which rules out the possibility of "open" tenants:

\section{A4 If $x$ is a niche for $y$, then $y$ is closed (i.e., its parts include its boundaries).}

This is motivated by our ecological interpretation of the niche-tenant relationship: a tenant is an organism or a population of organisms, so its boundaries are its surfaces (the outer layers of the organism's skins) which face out toward the niche. We take it that every topologically closed entity has an interior (has divisible bulk). It follows, therefore, that no niche can be a mere boundary. Since niches are externally in contact with their tenants (A2), a niche must always be open in the region in which it makes contact with its tenant (for, as we have seen, where two entities are externally connected, one must be open and the other closed). Indeed, if niches were mere boundaries, they would be parts of their tenants by A4, and this would contradict A1. 
An immediate corollary is that the tenant of a niche cannot itself be a niche, which in turn implies that the niche-tenant relationship is not only irreflexive but fully asymmetric. More generally, it follows that niches cannot themselves be niched. This does not exclude an organism from being such as to constitute a niche or natural setting for another entity, for example a micro-organism inside a human body. What it does rule out is that the hosting organism might serve this hosting function by itself. To see what is at issue here, note that, if every organism is topologically closed and every niche open (in the relevant contact area), then it follows that a microorganism lodged inside your body as a niched entity is not topologically connected to your body: there must be some distance between them, however small. The niche for the micro-organism is thus not your body itself (which is closed), nor a proper part thereof, but rather an entity including also the area immediately surrounding the micro-organism and separating the latter from you. (Clearly, this presupposes that your body has inner cavities, and therefore that it is not compact in the sense that we are here assuming to hold of all tenants. But this assumption will eventually be relaxed in the sequel.)

Our next axiom imposes on niches and their occupants a common constraint of topological regularity:

\section{A5 If $x$ is a niche for $y$, then $x$ and $y$ are both regular.}

An open entity $x$ is said to be regular, topologically, if it coincides with the interior of its own closure, i.e., of the entity obtained from $x$ itself by adding its boundary; and a closed entity $y$ is said to be regular if it coincides with the closure of its own interior, i.e., of the entity obtained from $y$ by removing its boundary. Thus, a regular object is, roughly speaking, an object which does not possess outgrowing boundary spikes, does not lack a single interior point, does not consist of two or more voluminous parts connected by interiorless filaments, and so on. The point of this axiom, then, is to exclude from the orbit of our theory niches and tenants with strange topologies, for example space-filling curves, deleted Tychonoff corkscrews, and other monsters. There are, to be sure, organisms that have a quasi-fractal structure (sponges, mosses) and niches whose porosity is important to their ecological role. The hole-part structure of such entities is enormously complex; they are nonetheless, like all entities falling within the province of biological science, regular in the sense at issue here.

Our last axiom says that niches are exclusive environmental contexts: they cannot be shared by distinct entities (though distinct entities may have overlapping niches, both in the mereological and in the spatial sense of 'overlap').

\section{A6 If $x$ is a niche for $y$ and also for $z$, then $y$ and $z$ are identical.}

Consider the inside of an ant's nest. This is, no doubt, an ecological niche for a clutch of eggs when they are laid (a disconnected tenant). But is it not also a niche for each separate egg? To see why this is not so, consider that the surrounding environment of each individual egg includes, or is determined by, the boundaries of its neighbors. The surface layout of the collective niche is quite different from the surface layout of the niche for each egg taken individually. Similar considerations apply in relation to a pair 
of twin fetuses inside a mother's womb. Each fetus helps to determine the niche for its neighbor. The womb as a whole serves as niche for the twinned pair.

\section{Remarks and Refinements}

The elementary apparatus defined by A1-A6 identifies what we regard as the basic (synchronic) theory of niches, understood as ecological contexts. A few comments are in order, at this point.

First, note that our axioms suggest that for every niche there is a tenant (as is clear from the use of a relational predicate, ' $x$ is a niche for $y$ '), but not that every organism or population is always in a niche. A diver crossing the boundary between water and air is arguably not in a niche but rather moving from one niche to another. The issues raised by cases such as this, however, are part and parcel of the general problem of motion and change, which goes beyond the limits of the purely synchronic framework presented here.

Second, our axioms do not guarantee that niches are closed under the basic mereological operations of fusion and product. If an object has two niches, their fusion need not be a niche, for it might lack the sort of homogeneity by which niches are typically characterized. Likewise, if an object has two niches, their intersection need not be a niche. Consider a group of cows in the middle of a large field with a water tank at each of the two extremities A and B. The whole field is a niche for the cows, as is the middle plus A and the middle plus B. But the intersection of the latter is not a niche, since the cows need water. This asymmetry of behavior with regard to mereological operations is one respect in which the concept of niche deviates from the purely topological concept of neighborhood. But there are many other properties of neighborhoods whose analogues for niches have an uncertain status. For instance, should we assume that every two niches of the same tenant have a common part? That every niche for a given tenant has a proper part which is itself a niche for that tenant? That every niche has a compact part which is itself a niche for the same tenant (a niche with no internal holes except those occupied by the tenant)?

Third, note that our axioms do not imply that niches are dissective: a niche for an entity $y$ may have proper parts that are not niches for $y$, even if those proper parts fully surround $y$. Thus, for instance, no non-regular proper part of a niche ever qualifies as a niche. Our axioms do not imply, either, that niches may be arbitrarily large. Thus, in particular, the mereological complement of an organism (the result of imagining the organism as having been deleted from the remainder of the universe) need not be a niche, according to the axioms here listed. (The axioms do not however rule this out. Indeed, a straightforward consistency proof for A1-A6 can be obtained precisely by taking ' $x$ is a niche for $y$ ' to hold only if $x$ is the complement of $y$.) There is in fact a problem of vagueness here. For what can be said about the outer boundaries of niches? In some cases the surface layout of the surrounding physical environment provides an upper limit to the niche extension (the worm in its wormhole, the scholar in her cell). In other cases, however (the fish in the ocean, the bird in the sky), no such physical limit may be provided: the outer boundary of the relevant niche is then 
in some sense vague, though we leave it open here whether this vagueness is ontological [34] or merely conceptual [18]. (This alternative is not peculiar to the vagueness of niches and arises in connection with most entities countenanced by ordinary discourse: what are the boundaries of a cloud? of a hurricane? of a mountain?)

Let us, finally, consider the question of the inner boundaries of ecological contexts- the boundaries that niches share with their tenants. As we have already pointed out, our axioms assume that tenants involve no internal cavities. However, this assumption is for complex organisms too strong. Thus if niches are to be self-connected (by A3) and tenants closed (by A4), this means that for complex organisms A2 will in general fall short of capturing the relevant sense in which a niche and its tenant are connected: the boundary around a tenant's inner cavity cannot be connected to an exterior niche. Difficulties arise even in cases where the tenant has a connected boundary. Topologically, all animals are doughnut-shaped; yet it hardly seems reasonable to suppose that every niche of John would snake through his digestive tract (though we may naturally suppose that the finger through Mary's wedding ring is part of the ring's niche). To resolve these issues, we therefore need to amend axiom A2. Let the compact closure of an object be the entity that results (intuitively) when we take the object together with those parts of its complement that lie on its inside and through its perforations - the mereological sum of the object together with all its holes [36]. It can be verified that the compact closure of a closed, regular object is always closed and regular. Accordingly, we may reformulate $\mathrm{A} 2$ as follows:

\section{A2'. If $x$ is a niche for $y$, then $x$ is connected to every tangential part of the compact} closure of $y$.

There is, to be sure, a further complication here. Typically a hole in an organism is a genuine hole, analogous to the hole inside a wedding ring. But there seem to be cases where the putative hole is, in virtue of the intimate causal interconnection of processes on either side of its boundary, analogous to an organ within the interior of the organism in question. And there are also some mixed cases, perhaps of the sort illustrated by the womb conceived abstractly as dilation in the uterine tract. If we allow for such possibilities, then A1 must be amended too, to allow for the possibility of spatial—though not mereological—overlap between a niche and its tenant. (The solution to this problem may well be a question of granularity: when viewed from the microscopic level, it seems, more holes become visible within the organism, and thus also more possibilities for the hosting of interior tenants.)

\section{Open Issues}

There are many issues left open by the theory of niches qua ecological contexts presented above.

One family of issues arises out of the fact, already mentioned above, that A1-A6 provide only a synchronic account: we would still need to introduce the important factors of dynamics and change, and above all to address the issue of the identity of niches and niched objects over time. We also need to address issues relating to the 
movement and interaction of organisms within and between their respective niches. We need to find a place for the special types of causal integrity that characterize niches and niched entities, and for the special types of niche assembly-structure that arise for example when groups of individuals collaborate. And we need to consider the question of how the niches for given objects are determined by the properties of their surroundings. What determines the shape and size of a niche? How do animal niches in this respect relate to those of organisms of other types, for example corals or crustaceans?

A second important family of problems relates to the question of the status of niches when tenants are absent. As we have seen, the use of a relational predicate, ' $x$ is a niche for $y$ ' suggests that every niche has a tenant. But are niches essentially dependent entities, as Lewontin [21] would have it? Are they merely generically dependent on their tenants in the same sense in which a hole is dependent on its material host, or a boundary on the voluminous body that it bounds? Do we need to distinguish different types of niche, some of which will survive the temporary or permanent departure or replacement of their tenants?

Further questions concern the patterns of interaction between different niches, or between niches of different sorts. What is the relation between my niche and your niche when you occupy a position within my niche and I within yours? What is the relation between my niche and yours when we are in conflict, for example when we compete for occupation of a given territory, or when you are predator and I am prey, or when we interact symbiotically? What, finally, is the biologically very important relation between the individual or token niche or habitat of a single organism or population of organisms and the niche-type of the corresponding species?

Some of these questions arise specifically with reference to the ecological notion of niche on which we have focused in the foregoing. But some have a more general status, and concern the notion of an ecological context at large, including its ramifications into other domains such as economics, anthropology, evolutionary biology, or even the theory of network security (see again [4]). The formal theory outlined in the above will, we hope, provide at least a starting point for providing answers to these questions.

\section{Appendix: Formal Matters}

In [30] the niche-tenant relation is axiomatized as a first-order theory on the basis of a simple apparatus built around three primitive relations: the mereological relation $\mathrm{P}(x, y)$ (" $x$ is part of $y$ "); the topological relation $\mathrm{B}(x, y)$ (" $x$ is a boundary for $y$ "); and the locative relation $\mathrm{L}(x, y)$ (" $x$ is located exactly at $y$ "). (See [9] for a study of the axiomatic principles governing these relations.) As it turns out, this is sufficient to axiomatize a fourth primitive, $\mathrm{N}(x, y)$, corresponding to the relation " $x$ is a niche for $y$ ", in a way that conforms to principles A1-A6 above. However, the formalization of our A1 in terms of the three basic primitives gives rise to some problems. A better account can be given if we allow ourselves another basic primitive, $\mathrm{H}(x, y)$, to be understood as expressing the relation " $x$ is a hole in $y$ ". Using this primitive (axioma- 
tized as in [6]), it is easy to define the notion of an interior hole, or cavity, and consequently the notion of one object (a niche) surrounding another (a tenant):

$\mathrm{D} 1 \quad \mathrm{IH}(x, y)=_{\mathrm{df}} \mathrm{H}(x, y) \wedge \forall z(\mathrm{~B}(z, y) \rightarrow \mathrm{B}(z, x)) \quad$ interior hole

$\mathrm{D} 2 \quad \mathrm{~S}(x, y)=_{\mathrm{df}} \exists z \exists u \exists w(\mathrm{IH}(z, x) \wedge \mathrm{L}(z, u) \wedge \mathrm{L}(y, w) \wedge \mathrm{P}(w, u)) . \quad$ surrounding

Accordingly, we can formalize A1 as follows:

$\mathrm{A} 1 * \quad \mathrm{~N}(x, y) \rightarrow \mathrm{S}(x, y)$.

As for A2, we need the auxiliary notions of connection and tangential parthood, whose ordinary characterization is as follows:

$\begin{array}{llr}\mathrm{D} 3 & \mathrm{C}(x, y)=_{\mathrm{df}} \mathrm{O}(x, y) \vee \exists z(\mathrm{P}(z, x) \wedge \mathrm{B}(z, y) \vee \mathrm{P}(z, y) \wedge \mathrm{B}(z, x)) & \begin{array}{r}\text { connection } \\ \text { tangential part }\end{array} \\ \mathrm{D} 4 & \mathrm{TP}(x, y)=_{\mathrm{df}} \mathrm{P}(x, y) \wedge \exists z(\mathrm{C}(x, z) \wedge \neg \mathrm{O}(z, y)) .\end{array}$

A2 can then be formalized as

$\mathrm{A} 2 * \quad \mathrm{~N}(x, y) \rightarrow \forall z(\mathrm{TP}(z, y) \rightarrow \mathrm{C}(z, x))$.

The more general version, A2', can be formalized in a similar fashion, using the operator of compact closure $\mathrm{k}$ as defined in [30]:

$\mathrm{A} 2^{\prime *} \mathrm{~N}(x, y) \rightarrow \forall z(\mathrm{TP}(z, y) \rightarrow \mathrm{C}(x, \mathrm{k}(z)))$.

Alternatively, we can rely on a stricter notion of surrounding:

D5 $\operatorname{ES}(x, y)=_{\mathrm{df}} \exists z \exists u(\mathrm{IH}(z, x) \wedge \mathrm{L}(z, u) \wedge \mathrm{L}(y, u))$. exact surrounding

Then $\mathrm{A} 1 *$ and $\mathrm{A} 2{ }^{\prime} *$ can be fused into a single axiom:

$\mathrm{A} 1^{\prime *} \mathrm{~N}(x, y) \rightarrow \mathrm{ES}(x, \mathrm{k}(y))$.

The formalization of the remaining axioms A3-A6 then proceeds exactly as in [30] (see axioms A17-A21 therein).

\section{Acknowledgments}

Support of the National Science Foundation through award BCS-9975557 from the Geography and Regional Science program is gratefully acknowledged.

\section{References}

1. Barker, R. G., 1968, Ecological Psychology. Concepts and Methods for Studying the Environment of Human Behavior, Stanford: Stanford University Press.

2. Barker, R. G., and Associates, 1978, Habitats, Environments, and Human Behavior, San Francisco: Jossey-Bass Publishers.

3. Bruggeman, J. P., 1996, Formalizing Organizational Ecology. Logical and Mathematical Investigations in Organization Theory, Dissertation, Center for Computer Science in Organization and Managenemt, University of Amsterdam. 
4. Cardelli, L., and Gordon, A. D., 1998, 'Mobile Ambients', in M. Nivat (ed.), Foundations of Software Science and Computational Structures. Proceedings of the First International Conference, Berlin and Heidelberg: Springer-Verlag, pp. 140-155.

5. Cartwright, R., 1975, 'Scattered Objects', in K. Lehrer (ed.), Analysis and Metaphysics, Dordrecht: Reidel, pp. 153-171.

6. Casati, R., and Varzi, A. C., 1994, Holes and Other Superficialities, Cambridge, MA, and London: MIT Press (Bradford Books).

7. Casati, R., and Varzi, A. C., 1996, 'The Structure of Spatial Location', Philosophical Studies 82, 205-239.

8. Casati, R., and Varzi, A. C., 1997, 'Spatial Entities', in O. Stock (ed.), Spatial and Temporal Reasoning, Dordrecht, Boston, and London: Kluwer Academic Publishers, pp. 73-96.

9. Casati, R., and Varzi, A. C., 1999, Parts and Places: The Structures of Spatial Representation, Cambridge, MA, and London: MIT Press (Bradford Books).

10. Cohn, A. G., and Varzi, A. C., 1998, 'Connection Relations in Mereotopology', in H. Prade (ed.), Proceedings of the 13th European Conference on Artificial Intelligence, Chichester: John Wiley \& Sons, pp. 150-154.

11. Cohn, A. G., and Varzi, A. C., 1999, 'Modes of Connection', in C. Freksa (ed.), Spatial Information Theory. Proceedings of the Fourth International Conference, Berlin and Heidelberg: Springer-Verlag, in press.

12. Damuth, J., 1985, "Selection among "Species": A Formulation in Terms of Natural Functional Units', Evolution 39, 1132-1146.

13. Eldredge, N., 1989, Macroevolutionary Dynamics: Species, Niches, and Adaptive Peaks, New York: McGraw-Hill.

14. Elton, C., 1927, Animal Ecology, New York: Macmillan.

15. Freeman, J., and Hannan, M. T., 1983, 'Niche Width and the Dynamics of Organizational Populations', American Journal of Sociology 88, 1116-1145.

16. Gibson, J. J., 1979, The Ecological Approach to Visual Perception, Boston: Houghton-Mifflin.

17. Hall, E. T., 1966, The Hidden Dimension, Garden City: Doubleday.

18. Heller, M., 1996, 'Against Metaphysical Vagueness', Philosophical Perspectives 10, 177-186.

19. Hutchinson, G. E., 1978, An Introduction to Population Ecology, New Haven: Yale University Press.

20. Johansson, I., 1998, 'Pattern as an Ontological Category', in N. Guarino (ed.), Formal Ontology in Information Systems, Amsterdam: IOS Press, pp. 86-94.

21. Lewontin, R., 1979, 'Sociobiology as an Adaptationist Program', Behavioral Science $24,5-14$.

22. Looijen, R. C., 1995, 'On the Distinction Between Habitat and Niche, and Some Implications for Species' Differentiation', in T. A. F. Kuipers and A. R. Mackor (eds.), Cognitive Patterns in Science and Common Sense, Amsterdam and Atlanta: Rodopi, pp. $87-108$

23. Milne, G. R., 1990, An Ecological Niche Theory Approach to the Assessment of Brand Competition in Fragmented Markets, Dissertation, School of Business Administration, University of North Carolina at Chapel Hill.

24. Sack, R. D., 1986, Human Territoriality. Its Theory and History, Cambridge: Cambridge University Press.

25. Schoggen, P., 1989, Behavior Settings. A Revision and Extension of Roger G. Barker's Ecological Psychology, Stanford: Stanford University Press.

26. Smith, B., 1995, 'On Drawing Lines on a Map', in A. U. Frank and W. Kuhn (eds.), Spatial Information Theory. A Theoretical Basis for GIS, Berlin and Heidelberg: Springer-Verlag, pp. 475-84.

27. Smith, B., 1996, 'Mereotopology: A Theory of Parts and Boundaries', Data and Knowledge Engineering 20, 1996, 287-304. 
28. Smith, B., 1997, 'Boundaries: An Essay in Mereotopology', in L. H. Hahn (ed.), The Philosophy of Roderick Chisholm (Library of Living Philosophers), Chicago and La Salle, IL: Open Court, pp. 534-61.

29. Smith, B., 1999, 'Objects and Their Environments: From Aristotle to Ecological Psychology', in A. Frank (ed.), The Life and Motion of Socioeconomic Units, London: Taylor and Francis, in press.

30. Smith, B., and Varzi, A. C., 1999a, 'The Niche', Noûs 33, 198-222.

31. Smith, B., and Varzi, A. C., 1999b, 'Fiat and Bona Fide Boundaries', Philosophy and Phenomenological Research, in press.

32. Sterelny, K., and Griffiths, P., 1999, Sex and Death: An Introduction to the Philosophy of Biology, Chicago: University of Chicago Press.

33. Taylor, R. B., 1988, Human Territorial Functioning. An Empirical, Evolutionary Perspective on Individual and Small Group Territorial Cognitions, Behaviors and Consequences, Cambridge: Cambridge University Press.

34. Tye, M., 1990, 'Vague Objects', Mind 99, 535-557.

35. Varzi, A. C., 1996a, 'Parts, Wholes, and Part-Whole Relations: The Prospects of Mereotopology,' Data and Knowledge Engineering 20, 259-86.

36. Varzi, A. C., 1996b, 'Reasoning about Space: The Hole Story', Logic and Logical Philosophy 4, 3-39.

37. Varzi, A. C., 1997, 'Boundaries, Continuity, and Contact', Noûs 31, 26-58.

38. Varzi, A. C., 1998, 'Basic Problems of Mereotopology', in N. Guarino (ed.), Formal Ontology in Information Systems, Amsterdam: IOS Press, pp. 29-38.

39. Whittaker, R. H., and Lewin, S. A. (eds.), 1975, Niche Theory and Applications (Benchmark Papers in Ecology 3), Stroudsburg, PA: Dowden, Hutchinson, and Ross. 